\title{
The ability to determine the non-transferrin-bound iron and total iron in the human placenta using high-performance liquid chromatography method
}

\author{
Corresponding author: \\ Dr. Marcin Koba \\ A. Jurasza 2 St. \\ 85-094 Bydgoszcz \\ Poland \\ e-mail: kobamar@cm.umk.pl \\ Phone/Fax: (48) (52) 5853942
}

Medical Research Journal 2017;

Volume 2, Number 4, 181-185

10.5603/MRJ.2017.0026

Copyright (C) 2017 Via Medica

ISSN 2451-2591

\begin{abstract}
Iron is one of the most important microelements in the human body. It is a component of haemoglobin, which transports oxygen to all cells in the organism. It is also used in the synthesis of myelin, neurotransmitters, and DNA and transfers electrons in biochemical reactions. Iron is also responsible for regular development of the foetus' central nervous system. Furthermore, as a result of Fenton reactions, iron leads to formation of toxic free radicals. The existence of non-transferrin-bound iron (NTBI) and its part desferrioxamine-chelatable iron ( $\mathrm{DCl}$ ) can be used to assess this element in the body. The placenta is an organ transition that is formed during pregnancy in the female organism. It has a dense web of blood vessels in which dynamic exchange of blood between mother and foetus takes place. As a result, a fraction of NTBI may be present in the placenta. The main goal of this work was to develop a method for determining total iron and desferrioxamine-chelatable iron in solid tissues - the human placenta.
\end{abstract}

Key words: desferrioxamine chelatable iron (DCl), Desferal, ferrioxamine (FO), high-performance liquid chromatography (HPLC), placenta, total iron

Med Res J 2017; 2 (4): 181-185

\section{Introduction}

Iron is a necessary component for the body to function properly. This basic microelement plays an integral role in most biochemical and metabolic processes in the human organism. Iron is a substrate for heme synthesis, it participates in the oxidation-reduction, immune reaction, and DNA synthesis [1]. Biological functions of iron include transportation and detection of diatomic gases and electrons transfer. Therefore, the human organism needs iron to transport oxygen to tissues and for electron transport in the Krebs cycle [2]. Iron in the body may be present on two oxidation levels: $\mathrm{Fe}^{+2}$ and $\mathrm{Fe}^{+3}$. It is a central component of heme - prosthetic group in haemoglobin, myoglobin, peroxidase, catalase, cytochrome $\mathrm{C}$, and other proteins. Total metabolically active iron is contained in $70 \%$ in heme, $20 \%$ in ferritin, and $10 \%$ in enzymes, and only $0.15 \%$ of iron is bound to transferrin. As previously mentioned, iron is mainly bound to protein, which is associated with its functions in the organism. Furthermore, in the human body there is also another form of iron: non-transferrin-bound iron (NTBI). The appearance of NTBI and its component - desferrioxamine-chelatable iron (DCl) - can be used for rating this element in the body [3-4].

Iron is toxic when it is present in abundance, so tight regulation is very important to avoid overload or deficiency of iron. The human organism is not a developed mechanism that removes iron from the body when it is overloaded. Losses of this component take place through intestinal secretions, desquamated epithelium, and also during menstruation in women. Several current researchers indicate the participation of severe untreated iron overload in the pathogenesis of diseases such as fibrosis and cirrhosis of the liver, diabetes, hypothyroidism, hypogonadism, and cardiac 
arrhythmias. Currently researchers are focusing on analysing the effects of less pronounced grades of iron overload in the development of chronic diseases [2].

Iron is also responsible for the regular development of the foetus' central nervous system. It is directly involved in production and proper functioning of myelin and nerve fibres [9]. Prenatal iron deprivation can inhibit the synthesis of nervonic acid. This can result in abnormalities in myelination of the axons. Iron deficiency also causes reduced activity of cytochrome c oxidase and alterations in metabolism of dopamine, serotonin, and other neurotransmitters [9-10]. Previous studies suggest that iron deficiency in the initial period of life affects brain and behavioural functions that persist despite the use of iron [11]. The average iron content in a foetus weighting $3500 \mathrm{~g}$ in the third trimester of pregnancy is $7.5 \mathrm{mg} / 100 \mathrm{~g}$ body weight [10].

The damaging effect of NTBI is associated with its ability to take part in the Fenton reaction, which may result, among others, in cellular membrane damage and induce apoptosis [3]. Previous studies have reported that NTBI is not detected in the serum of the healthy population $[4,12]$. The group most vulnerable to disorders caused by NTBI are newborns. NTBI is an indicator of the severity of intrauterine oxidative processes and brain damage in newborns. Buonocore et al. demonstrated that an NTBI cord blood concentration higher than $15.2 \mu \mathrm{mol} / \mathrm{l}$ is the best and earliest marker of central nervous system damage in this group [5-6]. Determination of NTBI has the potential to be an efficient method in the diagnosis of diseases associated with iron accumulation. Another important finding was that inflammatory mediators do not affect NTBI level (in contrast to routinely determined parameters such as saturation of transferrin or ferritin concentration) [13]. The presence of the carbonyl group on albumin and $\alpha$-fetoprotein in newborns with high concentration of NTBI was revealed. This demonstrates the intensified process of reactive oxygen species formation. Previous studies confirm that premature infants have increased concentrations of NTBI and products of protein and lipid oxidation than infants born at term. NTBI was also found in the amniotic fluid during physiological pregnancy and in about $25 \%$ of healthy newborns' serum [14-15].

The placenta is an organ transition that is formed during pregnancy in the female organism. Probably the highest concentration of iron occurs in the placenta, where we observe a dense web of blood vessels and where intensive exchange of metabolites between mother and foetus takes place. For this reason, the fraction of NTBI may be present in the placenta. The iron level so far has been studied primarily in the serum. There are no studies on the level of iron in solid tissue such as placenta. Iron deficiency can cause premature birth, low birth weight, infant developmental delays, and even infant death [2,7-8]. The effects of excess iron concentration in the foetus are not well known yet. This study presented a method for determination of iron concentration in the placenta.

The main aim of this study was to develop a method for determining NTBI and total iron in solid tissues and verification of the presence of these substances in human placenta. Iron analysis has been made by high performance liquid chromatography (HPLC). The study took into consideration 13 placentas from physiological pregnancy.

\section{Experimental}

\section{Chemicals or Reagents}

Desferrioxamine (DFO) as Desferal ${ }^{\circledR}$ (Deferoxamini mesilas, N-[5-[3-[(5-aminopentyl)hydroxy-carbamoyl] propionamido]pentyl]-3-[[5-(N-hydroxyacetamido) pentyl] carbamoyl] propionohydroxamic acid monomethanesulphonate) was from Novartis International AG (Basel, Switzerland). HPLC-grade acetonitrile and water were from $\mathrm{POCH}$ (Gliwice, Poland), and Tris- $\mathrm{HCl}$ was from Sigma-Aldrich (Poznań, Poland).

\section{Apparatus}

A Shimadzu High-Performance Liquid Chromatography set (Kyoto, Japan) equipped with vacuum degasser (DGU-20A5), solvent pump (LC-20AD), autosampler (SIL-20AD), diode array detector (SPDM20A), column oven (CTO-20AC), communications bus module (CBM-20A), and LC Solution software (ver. 1.0.0.1 Kyoto, Japan) was used for complex HPLC analysis. An MPW-350R centrifuge (Med. Instruments Cooperative, Warsaw, Poland) was used for serum sample preparation. An HPLC octadecylsilane (C18) column Candeza CD-C18 150x4.6 mm i.d., with particle size $3.0 \mu \mathrm{m}$ (Imtakt Corporation, Kyoto, Japan) equipped with a pre-column analytical guard cartridge system KJO-4282 (Phenomenex, Torrance, CA, USA) were used for chromatographic separation.

Preparation of placenta samples for determination of total iron and iron chelated by desferrioxamine

Sample preparation is a particularly important step in the analysis of the placenta, and it differs from the process of preparation of other types of tissues such as serum. Before tissues were analysed by HPLC they required prior preparation composed of several steps. Initially the placenta was homogenised. The first stage of homogenisation was tissue grinding in a mortar. Next, homogenisation was performed in a liquid nitrogen atmosphere, until homogeneous mass was obtained. Then cellular structures were broken down using Tris- $\mathrm{HCl}$ buffer $(\mathrm{pH}=8.5)$ and a $10 \%$ solution of Triton X-100. Subsequently, material was centrifuged 


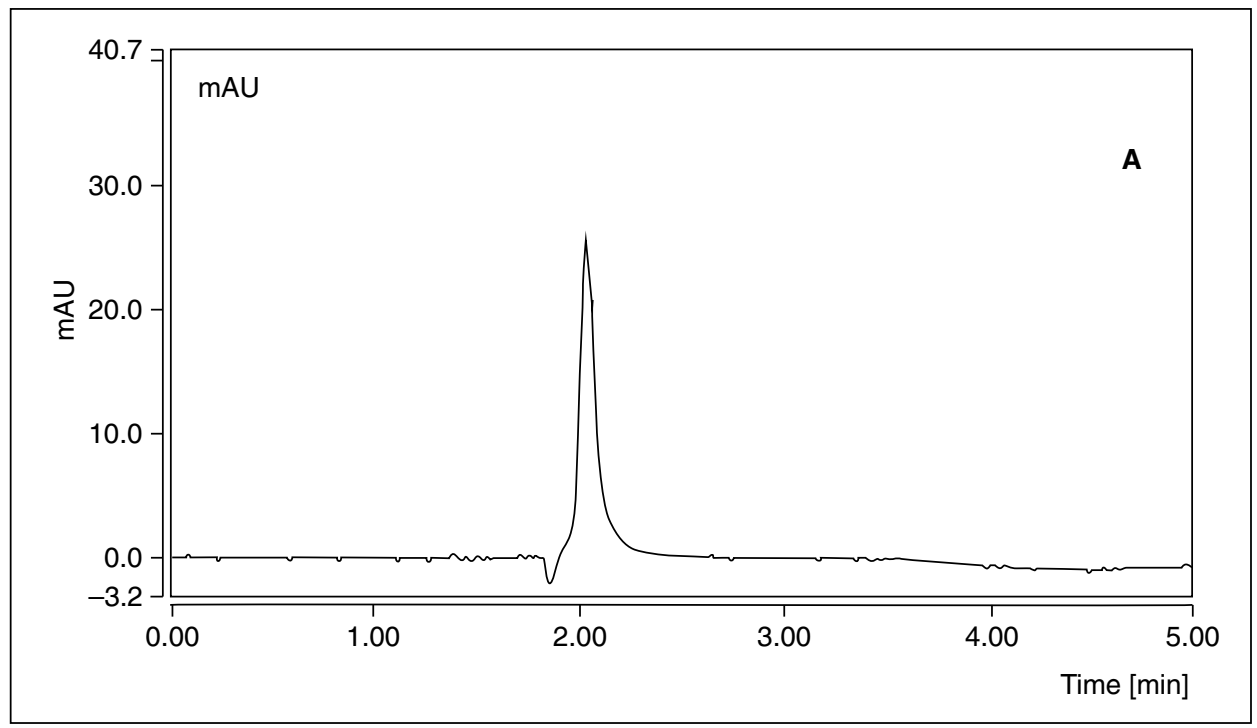

Figure 1. Representative chromatograms of total iron (150 $\mu \mathrm{M}$ concentration) on Cadenza column in $8.5 \mathrm{pH}$

and then the mixture was incubated in a refrigerator for $12 \mathrm{~h}$. After that time, samples were centrifuged and the supernatants of each sample were pipetted to Eppendorf. The next step was heating denaturation. This process resulted in the release of iron from proteins. For this purpose, samples were incubated in a thermocycler for 20 minutes at $90^{\circ} \mathrm{C}$. After this time, tubes were immediately transferred to ice for one minute. Next, the samples were centrifuged and the supernatants of each sample were pipetted to Eppendorf. Furthermore, the sample was purified on columns. The columns prevented impurities. After centrifugation the removed impurities and the resultant filtrate was used for further studies. The filtrate obtained from each placenta was mixed 1:1 with a solution of desferrioxamine. After standing for two hours in the dark, DCl complexes were formed. Chromatographic analysis was then performed on total iron and iron chelated by desferrioxamine in each of the placentas. To make results more reliable, some of the samples, before adding a solution of desferrioxamine, were supplemented with solutions of iron (III) at concentrations of $80 \mu \mathrm{M}$.

\section{Preparation of calibration curve}

The quantitative measurement was performed using the RP-HPLC method. Chromatographic separation was performed by gradient elution using a Cadenza CDC18 column with solvent $10 \mathrm{mM}$ Tris- $\mathrm{HCl}$ and solvent acetonitrile-ACN on the basis of a previously reported study by Koba et al. [3]. The step-form gradient elution conditions were optimised experimentally with a total run time of $15 \mathrm{~min}$ and with gradient changes in time of the HPLC analysis as follows: $15 \%$ during $0-1 \mathrm{~min}, 45 \%$ during 1-5 min, and 15\% during 5-15 min. The retention times of $\mathrm{FO}$ indicated on $\mathrm{DCl}$ or total iron concentration were repeatably near $2.1 \mathrm{~min}$ (for representative chromatograms see Figures 1 and 2).

\section{Results and discussions}

Human placenta is a solid tissue, and it is a challenge to use this as material for HPLC analysis. To ensure reliable and accurate measurements, proper sample preparation is very important. In reviewing the literature, no description of this process in solid tissue was found. Analyses in 10 samples of human placenta on a Cadenza C18 column were performed. All measurements were performed twice, and high repeatability was reported. Table 1 presents average values of all results. Table 1 presents the calculated concentrations of total iron in human placenta. The calculations are based on the equations of a calibration curve at $8.5 \mathrm{pH}$. Samples of placenta were supplemented with solutions of iron at concentrations of $80 \mu \mathrm{M}$ in order to make results more reliable. This allowed us to calculate a recovery [\%] and interpret the results or assess the accuracy of the method. Table 1 also presents the results of calculated total iron concentration after supplementation and calculated recovery.

Another parameter measurement in human placenta by HPLC was desferrioxamine-chelatable iron (DCl). It is a component of serum non-transferrin-bound iron (NTBI). Table 1 shows DCl concentrations calculated by calibration curve (average value). A previous study of ours indicated that $\mathrm{pH}$ changes affect the number of DCl-formed complexes. Therefore, the results of the 


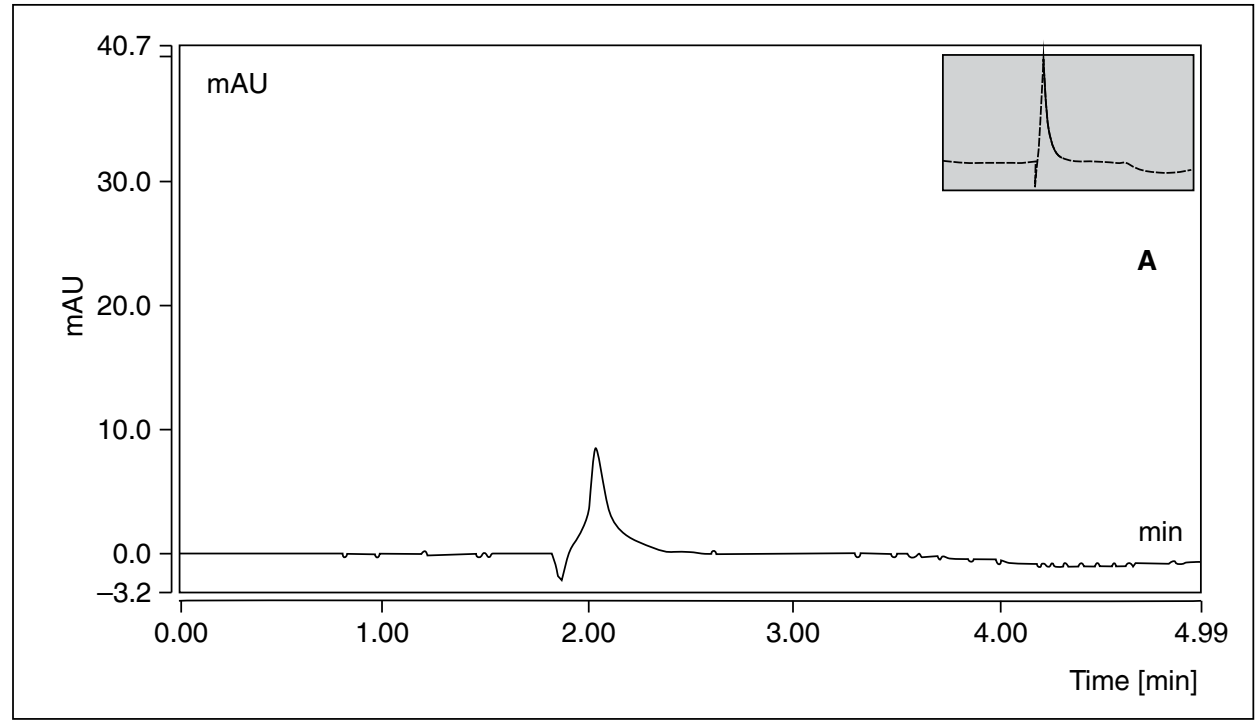

Figure 2. Representative chromatograms of $\mathrm{DCl}$ ( $50 \mu \mathrm{M}$ concentration) on Cadenza column in $8.5 \mathrm{pH}$.

Table 1. Results and recovery of total iron and $\mathrm{DCl}$ assays in placenta samples.

\begin{tabular}{|c|c|c|c|c|}
\hline Number & $\begin{array}{l}\text { Total iron concentration } \\
\text { in human placenta }[\mu \mathrm{M}]\end{array}$ & $\begin{array}{l}\text { Total iron supplemented } \\
(80 \mu \mathrm{M}) \text { concentration }[\mu \mathrm{M}]\end{array}$ & Recovery [\%] & $\begin{array}{c}\text { DCl concentration } \\
\text { in human placenta }[\mu \mathrm{M}]\end{array}$ \\
\hline 1 & 11.77 & 88.82 & $111 \%$ & 9.77 \\
\hline 2 & 13.21 & 88.56 & $111 \%$ & 5.25 \\
\hline 3 & 11.60 & 86.80 & $109 \%$ & 3.48 \\
\hline 4 & 11.45 & 89.07 & $111 \%$ & 2.42 \\
\hline 5 & 9.14 & 84.57 & $106 \%$ & 1.63 \\
\hline 6 & 12.71 & 89.47 & $112 \%$ & 8.92 \\
\hline 7 & 11.12 & 90.62 & $113 \%$ & 9.48 \\
\hline 8 & 10.66 & 90.56 & $113 \%$ & 4.31 \\
\hline 9 & 11.99 & 89.03 & $111 \%$ & 5.03 \\
\hline 10 & 11.25 & 87.19 & $109 \%$ & 3.65 \\
\hline
\end{tabular}

analysis should be related to a calibration curve with the same $\mathrm{pH}$ (in this study - $8.5 \mathrm{pH}$ ). Measurement on a Cadenza column demonstrated a high repeatability. Results of $\mathrm{DCl}$ concentration were in range of $1.6-9.8 \mu \mathrm{M}$ whereas total iron concentration ranged from $9.14 \mu \mathrm{M}$ to $13.20 \mu \mathrm{M}$.

As mentioned, in the literature review we noted a lack of data about norms of $\mathrm{DCl}$ and total iron concentration and about different methods of measurement of this parameter in human placenta. In this study, the presence of $\mathrm{DCl}$ and total iron in human placenta was demonstrated. All measurements were performed twice, and high repeatability was reported. The potential of application this method for sample preparation and analysis of $\mathrm{DCl}$ and total iron measurement in solid tissue after previous optimisation and improvement was presented. Therefore, the presented method may be used for routine assay of desferrioxamine-chelatable and/or total iron fractions in solid biological samples.

\section{References}

1. Papanikolaou G, Pantopoulos K. Iron metabolism and toxicity. Toxicol Appl Pharmacol. 2005; 202(2): 199-211, doi: 10.1016/j. taap.2004.06.021, indexed in Pubmed: 15629195.

2. Escobar-Morreale HF. Iron metabolism and the polycystic ovary syndrome. Trends Endocrinol Metab. 2012; 23(10): 509-515, doi: 10.1016/j. tem.2012.04.003, indexed in Pubmed: 22579050.

3. Koba M, Słomka A, Baczzek T, et al. Ability to determine the desferrioxamine-chelatable iron fractions of nontransferrin-bound iron using HPLC. J Sep Sci. 2013; 36(4): 665-669, doi: 10.1002/jssc.201200683, indexed in Pubmed: 23355397. 
4. Slomka A, Piotrowska K, Koba M, et al. Non-Transferrin Bound Iron - Determination in Biological Material and Clinical Implications. Current Pharmaceutical Analysis. 2011; 7(4): 280-285, doi: 10.2174/157341211797458014

5. Marzocchi B, Perrone S, Paffetti P, et al. Nonprotein-bound iron and plasma protein oxidative stress at birth. Pediatr Res. 2005; 58(6): 1295-1299, doi: 10.1203/01.pdr.0000183658.17854.28, indexed in Pubmed: 16306211.

6. Buonocore G. Perrone S, Longini M, et al. Non protein bound iron as early predictive marker of neonatal brain damage. Brain. 2003; 126(Pt 5): 1224-1230, indexed in Pubmed: 12690060.

7. Pazirandeh, S. et al. (2012) Overview of dietary trace minerals. Wolters Kluwer Health.

8. Johnson-Wimbley TD, Graham DY. Diagnosis and management of iron deficiency anemia in the 21st century. Therap Adv Gastroenterol. 2011; 4(3): 177-184, doi: 10.1177/1756283X11398736, indexed in Pubmed: 21694802.

9. Aggett PJ, Agostoni C, Axelsson I, et al. Iron metabolism and requirements in early childhood: do we know enough?: a commentary by the ESPGHAN Committee on Nutrition. J Pediatr Gastroenterol Nutr. 2002; 34(4): 337-345, indexed in Pubmed: 11930085
10. Słomka A, Zekanowska E, Piotrowska K, et al. Iron metabolism and maternal-fetal iron circulation. Postepy Hig Med Dosw. 2012; 66 : 876-887, doi: 10.5604/17322693.1019651.

11. Logan S, Martins S, Gilbert R. Iron therapy for improving psychomotor development and cognitive function in children under the age of three with iron deficiency anaemia. Cochrane Database Syst Rev. 2001(2) CD001444, doi: 10.1002/14651858.CD001444, indexed in Pubmed: 11405989.

12. Wang J, Pantopoulos K. Regulation of cellular iron metabolism. Biochem J. 2011; 434(3): 365-381, doi: 10.1042/BJ20101825, indexed in Pubmed: 21348856.

13. Słomka A, Włodarczyk W, Żekanowska E. Udział żelaza niezwiązanego z transferryną w patofizjologii chorób człowieka. Przegląd Med Uniw Rzesz i NIL w Warszawie Rzeszów. 2011; 1: 101-107.

14. Buonocore $\mathrm{G}$, Perrone $\mathrm{S}$, Longini $\mathrm{M}$, et al. Non protein bound iron as early predictive marker of neonatal brain damage. Brain. 2003; 126(Pt 5): 1224-1230, indexed in Pubmed: 12690060.

15. Dorrepaal CA, Berger HM, Benders MJ, et al. Nonprotein-bound iron in postasphyxial reperfusion injury of the newborn. Pediatrics. 1996 98(5): 883-889, indexed in Pubmed: 8909481. 